\title{
Künstliche Intelligenz (KI) in Zeiten des Datenschutzes
}

\section{Begriffseinordnung}

Künstliche Intelligenz (abgekürzt KI, oder englisch artificial intelligence, AI), ist ein Forschungsgebiet der Informatik, bei dem es um ein „intelligentes Problemlösungsverhalten“ sowie die Erstellung „intelligenter“ Computersysteme geht. ${ }^{1}$ Unterschieden wird dabei zwischen der Methode sowie der Anwendung „Künstlicher Intelligenz“ (KI). ${ }^{2}$ Beschrieben wird mit „Künstlicher Intelligenz“ zumeist der Versuch, menschenähnliche Entscheidungsstrukturen nachzubilden, so dass ein Computer Probleme vergleichbar einem Menschen zu lösen im Stande ist. Es werden aber auch Bereiche damit bezeichnet, in denen durch Algorithmen ein „intelligentes Verhalten“ simuliert werden soll, wie es beispielsweise bei Computerspielen der Fall ist. ${ }^{3}$

Maßstab für die Beurteilung des Vorliegens einer „Künstlichen Intelligenz" bleibt hingegen der für das menschliche Verhalten entwickelte Begriff der "Intelligenz" ${ }^{4}$ Schon aus diesem Grund bietet die konkrete Definition der Bezeichnung „Künstliche Intelligenz" einige Schwierigkeiten, fehlt es doch schon an einer einheitlichen Vorstellung der Einordnung „menschlicher Intelligen $z^{\text {" } 5}$

\section{Anwendungsbereiche}

„Künstliche Intelligenz“ findet sich inzwischen in ganz unterschiedlichen Bereichen wieder. ${ }^{6}$ Denkbare Einsatzmöglichkeiten bieten hierbei zum Beispiel sowohl Sprachassistenzsysteme ${ }^{7}$ als auch autonome Fahrzeugsysteme oder Systeme zur Unterstützung im Pflegebereich. ${ }^{8}$ Darüber hinaus werden jedoch vielfach zu Werbezwecken außerdem auch schlicht moderne Systeme -

1 Vgl. https://de.wikipedia.org/wiki/K\%C3\%BCnstliche_Intelligenz (letzter Abruf: 27.7.2018); https://wirtschaftslexikon.gabler.de/definition/kuenstliche-intelligenz-ki-40285 (letzter Abruf: 27.7.2018).

2 Dazu der Überblick unter https://wirtschaftslexikon.gabler.de/definition/ kuenstliche-intelligenz-ki-40285 (letzter Abruf: 27.7.2018); zu den Risiken schon Conrad, DuD 2017, S. 742.

3 Zu den Algorithmen siehe bei Wojak, DuD 2018, S. 553 (in diesem Heft).

4 Schael, DuD 2018, S. 547 (in diesem Heft).

5 Siehe hierzu unter Vgl. https://de.wikipedia.org/wiki/K\%C3\%BCnstliche_In telligenz (letzter Abruf: 27.7.2018); Schael, DuD 2018, S. 547 (in diesem Heft).

6 Conrad, DuD 2018, S. 541 (in diesem Heft); Rost, DuD 2018, S. 558 (in diesem Heft).

7 Rost, DuD 2018, S. 558 (in diesem Heft).

8 Schael, DuD 2018, S. 547 (in diesem Heft). wegen beispielsweise einer intelligenten Sprachsteuerung - ebenfalls mit dem Begriff „Künstliche Intelligenz" betitelt (vgl. zum Beispiel bei Cortana, Alexa, Siri und ok-Google). ${ }^{9}$

Doch unabhängig von der Frage der gerechtfertigten Bezeichnung als „Künstliche Intelligenz “, ist für den Datenschutz wesentlich, dass bei der Nutzung derartiger Systeme auf jeden Fall zahlreiche personenbezogene Daten im Sinne des Art. 4 Nr. 1 Datenschutz-Grundverordnung (DS-GVO) verarbeitet werden. ${ }^{10}$

\section{Datenschutz-Grundverordnung}

Bereits am 24. Mai 2016 trat die EU-Datenschutz-Grundverordnung (DS-GVO oder auch DSGVO, EU-DSGVO) in Kraft, die gemäß Art. 99 Abs. 2 S. 1 DS-GVO seit dem 25. Mai 2018 gilt und damit die Richtlinie 95/46/EG ablöst. ${ }^{11}$ Anders als die Richtlinie zum Datenschutz, muss die Verordnung nicht erst in nationales Recht umgesetzt werden, sondern ihre Regelungen gelten verbindlich und unmittelbar in allen EU-Mitgliedstaaten. ${ }^{12}$ Zwar enthält die Verordnung Regelungen, die dem nationalen Gesetzgeber die Möglichkeit geben eigene Gesetze zu erlassen (umgangssprachlich: sog. Öffnungsklauseln), diese dürfen jedoch nicht gegen die Regelungen bzw. Vorgaben der Datenschutz-Grundverordnung verstoßen. Im Zusammenhang mit dem Einsatz der „Künstlichen Intelligenz" stellen sich daher zahlreiche datenschutzrechtliche Fragen, die bereits bei der Klärung der Verantwortung im Sinne des Art. 4 Nr. 7 DS-GVO für die Verarbeitung von personenbezogenen Daten durch „Künstliche Intelligenz “ ansetzen. ${ }^{13}$ Darüber hinaus sind die sich aus der Datenschutz-Grundverordnung ergebenden Pflichten sowie Rechte der betroffenen Personen zu beachten, wozu unter anderem auch die Notwendigkeit einer Datenschutz-Folgenabschätzung (DSFA) nach Art. 35 DSGVO und die Umsetzung ausreichender technischer und organisatorischer Maßnahmen im Sinne des Art. 32 DS-GVO bei Einsatz „Künstlicher Intelligenz“ gehören. ${ }^{14}$

\footnotetext{
9 Vgl. Schael, DuD 2018, S. 547 (in diesem Heft).

10 Dazu ausführlich bei Conrad, DuD 2018, S. 541 (in diesem Heft).

11 Aktueller Text zu finden unter https://eur-lex.europa.eu/legal-content/ DE/TXT/?uri=celex\%3A32016R0679 (letzter Abruf: 27.7.2018).

12 Siehe auch Mester, DuD 2013, S. 250.

13 Wojak, DuD 2018, S. 553 (in diesem Heft).

14 Insgesamt hierzu bei Rost, DuD 2018, S. 558 (in diesem Heft).
} 